\title{
Endovascular procedures for treating wide-necked aneurysms
}

\author{
David Wells-Roth, M.D., Alessandra Biondi, M.D., Vallabh Janardhan, M.D., \\ Kyle Chapple, M.D., Y. Pierre Gobin, M.D., and Howard A. RiIna, M.D.
}

Departments of Neurology, Radiology, and Neurological Surgery, New York Presbyterian Hospital, Weill Medical College of Cornell University, New York, New York

\begin{abstract}
Wide-necked aneurysms remain difficult to treat by either open microneurosurgical or endovascular procedures. Recent advances in the latter technology, including intracranial stents and bioactive coils, now allow an endovascular treatment option for cases in which this was not previously available. In this report the authors describe the new developments in endovascular technologies that make the treatment of wide-necked aneurysms possible. This includes discussion of intracranial stents and bioactive coils designed to promote obliteration of the aneurysm lumen. In addition, methods for coil insertion in wide-necked aneurysms are described, including balloon remodeling and various stent placement procedures. Wide-necked aneurysms previously thought to be untreatable by endovascular means can now be obliterated, thanks to new devices specifically designed for intracranial use.
\end{abstract}

\section{KEY WORDS - wide-necked aneurysm - endovascular therapy - intracranial stent • bioactive coil - balloon remodeling}

\section{OVERVIEW}

In 1995, the Guglielmi detachable coil system received US Food and Drug Administration approval, which began the era of nonsurgical management of intracranial aneurysms. ${ }^{4}$ At that time, the majority of aneurysms that needed treatment were primarily considered to be amenable to surgery. In subsequent years, the criteria for endovascular treatment have broadened and, at many institutions, the endovascular approach is now considered an effective treatment in most cases for both ruptured and unruptured aneurysms.

Several angiographic and clinical features have been described for determining which aneurysms are most suitable for either an endovascular, open surgical, or combined treatment approach. These features include aneurysm location, size, morphology, neck size, and clinical status. ${ }^{2}$ Nevertheless, aneurysms with wide necks remain difficult to treat surgically and, in the same way, both wide-necked and geometrically difficult aneurysms have remained a challenge for endovascular therapy. Currently, thanks to advances in materials and microsurgical techniques, an endovascular treatment is possible in widenecked and fusiform aneurysms that could previously only be treated by surgical clip placement. Nevertheless, fusiform aneurysms have remained difficult to treat because by definition they have no neck. In fusiform aneurysms, an effective treatment is not always possible and occlusion of the parent artery may be required. Patients who do not tolerate parent vessel occlusion may require surgical bypass.

\section{TREATMENT OPTIONS}

The selective endovascular treatment of wide-necked aneurysms now involves a combination of many options that includes the following: 1) new types of coils; 2) balloon remodeling; and 3) intracranial stents.

\section{New Coil Types}

Advanced coil technology has contributed greatly to improvements in securing these lesions. New coil designs, including three-dimensional shapes, soft coils, and ultrasoft coils, have also contributed to our improved ability to obliterate aneurysms. In addition, since the advent of bare platinum coils, new bioactive devices have now become available. The latter include Matrix detachable coils (Boston Scientific, Fremont, CA), which combine a pure platinum backbone with an added biodegradable copolymer, and hydrogel-coated coils (HydroCoils; MicroVention, Aliso Viejo, CA), which swell to three times their original size. Recurrence of lesions is still a major limitation of therapy with bare coils. Anecdotal practical experience has supported the theory that aneurysm recanalization and regrowth are more likely in large or giant lesions, when the aneurysm has a neck diameter larger than $4 \mathrm{~mm}$, or the neck/fundus ratio is greater than or equal to $1: 2$. Bioabsorbable polymer-coated platinum coils and expanding coils may actually promote healing at the aneurysm neck, where recanalization or recurrence is most likely to occur. ${ }^{9}$

\section{Balloon Remodeling}

The development of the balloon remodeling method, 
which was first described by Moret, et al., ${ }^{7}$ allows a larger portion of the spectrum of aneurysms to be treated using endovascular means. In the treatment of widenecked aneurysms, one of the major risks is the possibility of coil herniation through the broad neck into the parent vessel. This can cause thromboembolic events, which are the most frequent and serious complications associated with endovascular treatment of intracranial aneurysms.

The remodeling procedure consists of inflating a soft balloon in the parent artery along the wide neck of the aneurysm while a coil is positioned and subsequently detached (Fig. 1). Therefore, the balloon assistance allows for the temporary remodeling of the aneurysm neck during coil delivery. This method can be used for widenecked aneurysms at almost any location. ${ }^{1}$ This procedure initially involves placing a microcatheter with a nondetachable balloon in the parent artery across the neck of the aneurysm. An additional microcatheter is positioned in the sac of the aneurysm, with the balloon deflated. This balloon is inflated prior to release of the coil, and then, before detachment of the coil, the balloon is carefully deflated so that the stability of the coil mass can be assessed. This process is repeated with the delivery of each successive coil (Fig. 1). The balloon serves two purposes during embolization; first to stabilize the microcatheter in the aneurysm during coil delivery, and second to force the coil to assume the three-dimensional shape of the aneurysm without impinging on the parent vessel. ${ }^{6}$ In addition, the balloon procedure as well as the newer-generation coils allows for increased packing density, which assists in accelerating thrombus formation.

The primary limitation of this method is the inability to access the parent vessel at the level of the aneurysm neck, preventing optimal positioning of the balloon microcatheter. A $10 \%$ failure rate of this procedure has been reported in the literature. ${ }^{7}$ Aletich, et al., ${ }^{1}$ and Moret, et al., ${ }^{7}$ who originally described this method in their series of 79 and 53 patients, respectively, both have reported a morbidity and mortality rate between 1 and $5 \%$ in patients who were treated with balloon-assisted coil insertion. The most notable risk entailed embolic phenomena.

\section{Intracranial Stent Assistance}

The introduction of intracranial stents has contributed significantly to the treatment options for coil occlusion of wide-necked and fusiform aneurysms. Stent-assisted coil embolization prevents protrusion of the device into the parent vessel. In addition, intracranial stents might help to prevent aneurysm recanalization, which can still be observed despite the use of bioactive coils and balloon remodeling techniques.

Initially, the use of coronary stents as an adjunct to coil embolization was considered. These balloon-mounted stents developed for the coronary vessels lack the suppleness necessary to navigate the tortuosities of the intracranial circulation and to reach lesions beyond the carotid siphon. Therefore, these devices could only be used for sidewall aneurysms on parent vessels that were accessible by the stent.

The first stent designed for intracranial use was the Neuroform device (Boston Scientific). This stent is constructed of nitinol (a nickel-titanium alloy that assumes a

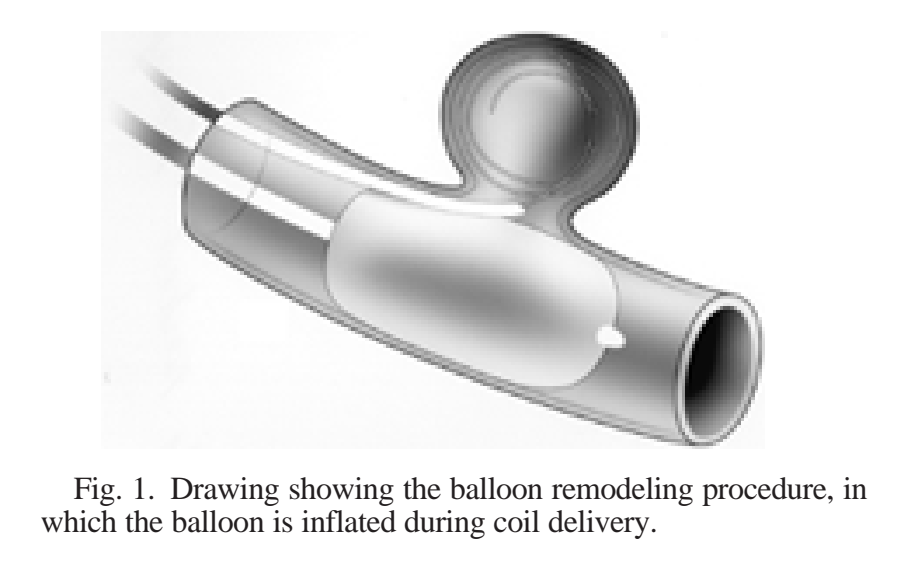

predetermined shape in appropriate conditions). This flexible material design enables the stent to be navigated within the tortuous intracranial vessels and to span the neck of a branch point effectively, which is where many intracerebral aneurysms occur. ${ }^{5}$

The stent-assisted coil delivery procedure consists of the placement of the stent in the parent artery across the aneurysm neck. A microcatheter is then navigated through the stent interstices into the aneurysm sac, and coil embolization is performed (Fig. 2). In a modified stent delivery procedure, the microcatheter is inserted into the aneurysm sac first, and then the stent is placed across the wide neck of the lesion. This "jails" the microcatheter in the aneurysm, helping to stabilize the device during delivery of the coils (Fig. 3). The disadvantage with this method is that there is the potential to move or even damage the stent when removing the microcatheter.

At our institution we perform what could be described as balloon remodeling-assisted stent placement. A widenecked aneurysm is occluded with coils by using the balloon remodeling procedure, leaving the final portion of the neck empty. Next, the stent is delivered and the remaining portion of the aneurysm neck is filled with coils. We believe that this adds a degree of safety to the procedure. Another strategy is that described by Howington, et al., ${ }^{5}$ consisting of a staged treatment. At the first endovascular session, only the stent is placed and the process of endothelialization (healing of the stent to the vessel wall) is allowed to occur. A second embolization session is later performed to achieve coil occlusion of the aneurysm sac.

The stent serves as a mechanical scaffold for placement of the coils into the aneurysm. Stent placement can allow safe packing of the lesion, preventing herniation of the coils into the parent vessels and allowing for a denser coil mesh. The increased resistance to high flow into the aneurysm dome because of the stent interstices is partly responsible for reduced intraaneurysm flow vortices and increased stasis. ${ }^{8}$ An additional advantage may be the reestablishment of laminar flow patterns, which actually may heal the aneurysm at its neck. This advantage may reduce the risk of future coil compaction, which is often observed in wide-necked aneurysms.

Technical problems with stent delivery have been reported in the literature. For example, Fiorella, et al., ${ }^{3}$ reported six cases in which difficulties with stent delivery were encountered. Additionally, in two other cases, the 


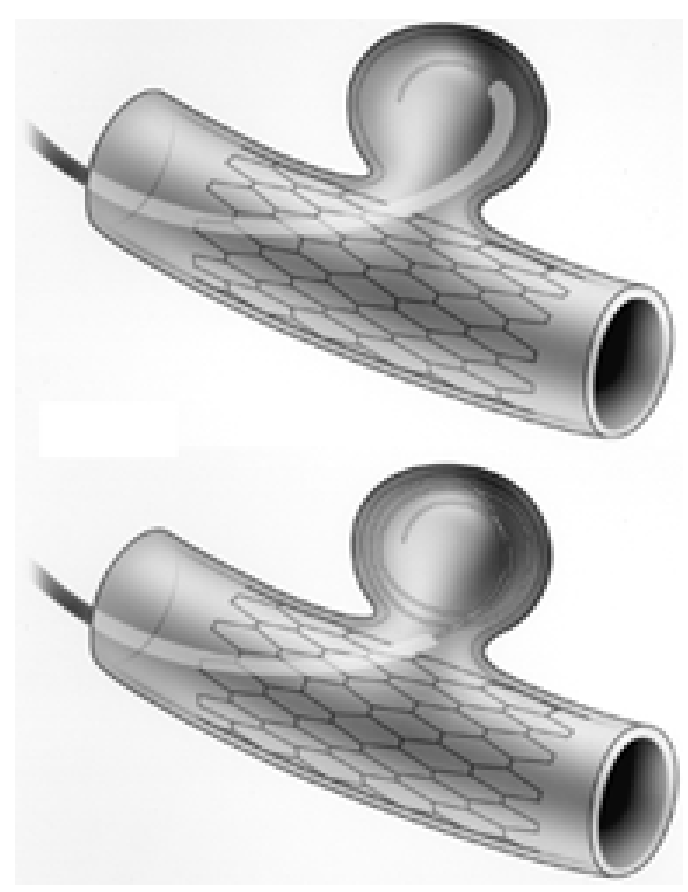

Fig. 2. Drawings showing the stent-assisted coil insertion procedure, in which the microcatheter is positioned through the stent prior to coil delivery.

same authors reported stent displacement after delivery during microcatheter manipulation. Newer stents have increased radial force and are more easily positioned and deposited. A new generation of the Neuroform stent has additional cross-links, allowing increased radial force, and can also be used for intracranial stenosis. This new selfexpanding stent has an open-cell mesh design, similar to its predecessor. It exerts a lower radial force than the coronary stents used for atherosclerotic disease, and is specifically designed for intracranial structures.

Although intracranial stents represent a significant advance in the endovascular treatment of wide-necked intracranial aneurysms, a disadvantage is that these devices are very thrombogenic. An intravascular stent stimulates platelet aggregation as soon as it is exposed to the patient's blood. It is currently recommended that patients re-

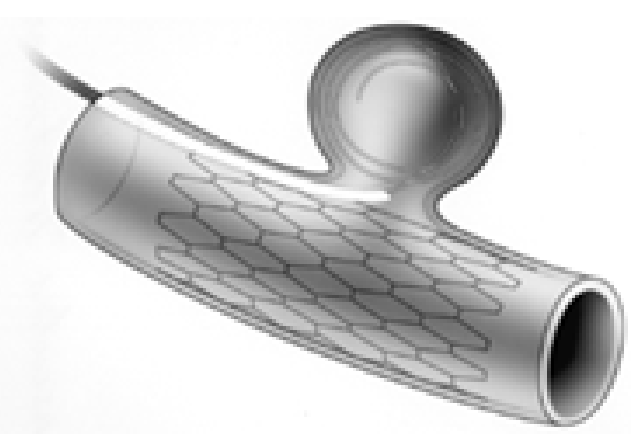

Fig. 3. Drawing showing the stent jailing method, in which the microcatheter is jailed between the vessel wall and the stent prior to coil delivery. ceive dual antiplatelet therapy 3 days before stent placement. Consequently, the ideal aneurysm for stent-assisted coil placement is an unruptured one, and such a lesion is treated effectively after appropriate antiplatelet therapy has been initiated. Although the antiplatelet regimen is contraindicated in patients with an acutely ruptured aneurysm, some options can be considered in a ruptured lesion that might benefit from stent-assisted coil placement. In such cases, an alternative is to perform coil occlusion (usually with the balloon remodeling procedure), and then later to repeat treatment of the residual neck with a stent.

\section{CONCLUSIONS}

Wide-necked and geometrically difficult aneurysms continue to be a challenge for both endovascular therapy and open microsurgery. New technology and devices continue to improve our ability to treat complex vascular lesions effectively by using endovascular means. The introduction of intracranial stents and bioactive coils now allows us to treat these lesions safely and effectively by an endovascular route.

\section{References}

1. Aletich VA, Debrun GM, Misra M, et al: The remodeling technique of balloon-assisted Guglielmi detachable coil placement in wide-necked aneurysms: experience at the University of Illinois at Chicago. J Neurosurg 93:388-396, 2000

2. Boulos AS, Levy EI, Bendok BR, et al: Evolution of neuroendovascular intervention: a review of advancement in device technology. Neurosurgery 54:438-453, 2004

3. Fiorella D, Albuquerque FC, Han P, et al: Preliminary experience using the Neuroform stent for the treatment of cerebral aneurysms. Neurosurgery 54:6-17, 2004

4. Henkes H, Fischer S, Weber W, et al: Endovascular coil occlusion of 1811 intracranial aneurysms: early angiographic and clinical results. Neurosurgery 54:268-285, 2004

5. Howington JU, Hanel RA, Harrigan MR, et al: The Neuroform stent, the first microcatheter-delivered stent for use in the intracranial circulation. Neurosurgery 54:2-5, 2004

6. Lefkowitz MA, Gobin YP, Akiba Y, et al: Balloon-assisted Guglielmi detachable coiling of wide-necked aneurysms: Part II-clinical results. Neurosurgery 45:531-538, 1999

7. Moret J, Cognard C, Weill A, et al: [Reconstruction technic in the treatment of wide-neck intracranial aneurysms. Long-term angiographic and clinical results. Apropos of 56 cases.] J Neuroradiol 24:30-44, 1997 (Fre)

8. Phatouros CC, Sasaki TY, Higashida RT, et al: Stent-supported coil embolization: the treatment of fusiform and wide-neck aneurysms and pseudoaneurysms. Neurosurgery 47:107-115, 2000

9. Regli L, Uske A, de Tribolet N: Endovascular coil placement compared with surgical clipping for the treatment of unruptured middle cerebral artery aneurysms: a consecutive series. J Neurosurg 90:1025-1030, 1999

Manuscript received December 22, 2004

Accepted in final form January 6, 2005.

Address reprint requests to: Howard A. Riina, M.D., Department of Neurological Surgery, New York Presbyterian Hospital, Weill Medical College of Cornell University, 525 East 68th Street, Starr 651, New York, New York 10021. email: HAR9005@med.cornell. edu. 